\title{
Dynamic behavior of a nonlinear rational difference equation and generalization
}

\author{
Qihong Shi', Qian Xiao, Guogiang Yuan and Xiaojun Liu
}

* Correspondence: shiqh03@163. com

Department of Basic Courses, Hebei Finance University, Baoding 071000, PR China

\begin{abstract}
This paper is concerned about the dynamic behavior for the following high order nonlinear difference equation $x_{n}=\left(x_{n-k}+x_{n-m}+x_{n-1}\right) /\left(x_{n-k} x_{n-m}+x_{n-m} x_{n-1}+1\right)$ with the initial data $\left\{x_{-l}, x_{-l+1}, \ldots, x_{-1}\right\} \in \mathbb{R}_{+}^{l}$ and $1 \leq k \leq m \leq l$. The convergence of solution to this equation is investigated by introducing a new sequence, which extends and includes corresponding results obtained in the references ( $\mathrm{Li}$ in J Math Anal Appl 312:103-111, 2005; Berenhaut et al. Appl. Math. Lett. 20:54-58, 2007; Papaschinopoulos and Schinas J Math Anal Appl 294:614-620, 2004) to a large extent. In addition, some propositions for generalized equations are reported.
\end{abstract}

Keywords: Nonlinear; Difference equation, Global stability, Positive solution

\section{Introduction}

Our aim in this paper is to study the dynamical behavior of the following equation

$$
x_{n}=\frac{x_{n-k}+x_{n-m}+x_{n-l}}{x_{n-k} x_{n-m}+x_{n-m} x_{n-l}+1}, \quad n=0,1,2, \ldots
$$

where the initial data $\left\{x_{-l}, x_{-l+1}, \ldots, x_{-1}\right\} \in \mathbb{R}_{+}^{l}$ and $1 \leq k \leq m \leq 1$.

The study of properties of similar difference equations has been an area of intense interest in recent years [1-3]. There have been a lot of work concerning the behavior of the solution. In particular, Çinar [4] studied the properties of positive solution to

$$
x_{n+1}=\frac{x_{n-1}}{1+x_{n} x_{n-1}}, \quad n=0,1, \ldots
$$

Yang et al. [5] investigated the qualitative behavior of the recursive sequence

$$
x_{n+1}=\frac{a x_{n-1}+b x_{n-2}}{c+d x_{n-1} x_{n-2}}, \quad n=0,1, \ldots,
$$

Li et al. [6] studied the global asymptotic of the following nonlinear difference equation

$$
x_{n+1}=\frac{x_{n-1} x_{n-2} x_{n-3}+x_{n-1}+x_{n-2}+x_{n-3}+a}{1+x_{n-1} x_{n-2}+x_{n-1} x_{n-3}+x_{n-2} x_{n-3}+a}, \quad n=0,1, \ldots
$$

with $a \geq 0$.

For more similar work, one can refer to [7-9] and references therein. Investigation of the equation (1.1) is motivated by the above studies. However, due to the special nonlinear relation, the methods mentioned in the references [4,5,7] do not always work for

(c) 2011 Shi et al; licensee Springer. This is an Open Access article distributed under the terms of the Creative Commons Attribution License (http://creativecommons.org/licenses/by/2.0), which permits unrestricted use, distribution, and reproduction in any medium, provided the original work is properly cited. 
the equation (1.1). In fact, equation (1.1) has lost the perfect symmetry. To this end, we introduce a simple transformed sequence to construct a contraction to prove the convergence of solutions, and apply this way solving a class of general equation.

The rest of this paper proceeds as follows. In Sect. 2, we introduce some definitions and preliminary lemmas. Section 3 contains the main results and their proofs. In Sect. 4 , we prove the stability for generalized rational difference equations and present our conjectures for similar equations.

\section{Preliminaries}

In this section, we introduce some basic but important preliminary lemmas and notation. For any $x_{i} \in \mathbb{R}_{+}$, we define a new sequence as $x_{i}^{*}=\max \left\{x_{i}, 1 / x_{i}\right\}$. With the help of the transformed sequence $\left\{x_{i}^{*}\right\}$, we can deduce the following conclusion.

Lemma 1. Suppose the function $f$ is defined by

$$
f(x, y, z)=\frac{x+y+z}{x y+y z+1}
$$

then $f$ is decreasing in $x$ and $z$ if and only if $y>1$ and increasing in $x$ and $z$ if and only if $y<1$. Similarly, $f$ is decreasing in $y$ if and only if $x+z>1$, conversely, it is increasing in $y$.

Proof. This conclusion follows directly from the fact

$$
\frac{\partial}{\partial x} f(x, y, z)=\frac{1-y^{2}}{(x y+y z+1)^{2}}
$$

and

$$
\frac{\partial}{\partial y} f(x, y, z)=\frac{1-(x+z)^{2}}{(x y+y z+1)^{2}}
$$

Since $x$ and $z$ is symmetrical, then the proposition is obvious.

Moreover, we can also prove the following contraction lemma which is useful in showing convergence of solutions in the transformed space mentioned in first paragraph of this section.

Lemma 2. Suppose $x_{n}$ satisfying the equation (1.1), for any $n \geq l$ and $\left(x_{n-k}, x_{n-m}, x_{n-l}\right) \in \mathbb{R}_{+}^{3}$, we have

$$
1 \leq x_{n}^{*} \leq x_{n-m}^{*} .
$$

Proof. Noticed that

$$
x_{n-k}+x_{n-m}+x_{n-l}-\left(1+x_{n-k} x_{n-m}+x_{n-m} x_{n-l}\right)=-\left(x_{n-m}-1\right)\left(x_{n-k}+x_{n-l}-1\right)
$$

and hence from (1.1), $x_{n} \leq 1$ whenever $x_{n-m}-1$ and $x_{n-k}+x_{n-l}$ - 1 are of the same signs, otherwise, $x_{n} \geq 1$. Let $x_{n-k}=u, x_{n-m}=v, x_{n-l}=w$. The RHS of (2.4) is obvious. Next we prove the LHS part. Indeed we have eight cases to consider. when $(1-v)(u+$ $w-1) \geq 0$, then

$$
x_{n}^{*}=x_{n}=\frac{u+v+w}{u v+v w+1} .
$$


Case (1) $(u \leq 1, v \leq 1, w \geq 1, u+w \geq 1)$. Here, by lemma 1 , note that $v^{*} \geq 1$, we have

$$
x_{n}^{*}=\frac{\frac{1}{u^{*}}+\frac{1}{v^{*}}+w^{*}}{1+\frac{1}{u^{*} v^{*}}+\frac{1}{v^{*}} w^{*}}=\frac{u^{*}+v^{*}+u^{*} v^{*} w^{*}}{1+u^{*} v^{*}+u^{*} w^{*}} \leq v^{*} .
$$

Case (2) $(u \geq 1, v \leq 1, w \leq 1, u+w \geq 1)$. Here, since $v^{*} \geq 1, w^{*} \geq 1$, we have

$$
x_{n}^{*}=\frac{u^{*}+\frac{1}{v^{*}}+\frac{1}{w^{*}}}{u^{*} \frac{1}{v^{*}}+\frac{1}{v^{*}} \frac{1}{w^{*}}+1}=\frac{w^{*}+v^{*}+u^{*} v^{*} w^{*}}{1+w^{*} u^{*}+v^{*} w^{*}} \leq v^{*} .
$$

Case (3) $(u \geq 1, v \leq 1, w \geq 1, u+w \geq 1)$. Similarly,

$$
x_{n}^{*}=\frac{u^{*}+\frac{1}{v^{*}}+w^{*}}{1+u^{*} \frac{1}{v^{*}}+\frac{1}{v^{*}} w^{*}}=\frac{u^{*} v^{*}+1+v^{*} w^{*}}{u^{*}+v^{*}+w^{*}} \leq v^{*} .
$$

Case (4) $(u \leq 1, v \geq 1, w \leq 1, u+w \leq 1)$. Here,

$$
x_{n}^{*}=\frac{\frac{1}{u^{*}}+v^{*}+\frac{1}{w^{*}}}{1+\frac{1}{u^{*}} v^{*}+v^{*} \frac{1}{w^{*}}}=\frac{u^{*}+w^{*}+u^{*} v^{*} w^{*}}{u^{*} v^{*}+u^{*} w^{*}+v^{*} w^{*}} \leq v^{*} .
$$

Oppositely, if $(1-v)(u+w-1) \leq 0$, from the definition of $x^{*}$, it is obvious that

$$
x_{n}^{*}=\frac{1}{x_{n}}=\frac{u v+v w+1}{u+v+w} .
$$

Case (5) $(u \leq 1, v \leq 1, w \leq 1, u+w \leq 1)$. By definition of $x_{n}^{*}$ Lemma 1 and the fact $v^{*} \geq 1$, we have

$$
x_{n}^{*}=\frac{1+\frac{1}{u^{*}} \frac{1}{v^{*}}+\frac{1}{v^{*}} \frac{1}{w^{*}}}{\frac{1}{u^{*}}+\frac{1}{v^{*}}+\frac{1}{w^{*}}}=\frac{u^{*}+w^{*}+u^{*} v^{*} w^{*}}{v^{*} w^{*}+u^{*} v^{*}+u^{*} w^{*}} \leq v^{*} .
$$

Case (6) $(u \geq 1, v \geq 1, w \leq 1, u+w \geq 1)$. Here, we have

$$
x_{n}^{*}=\frac{1+u^{*} v^{*}+v^{*} \frac{1}{w^{*}}}{u^{*}+v^{*}+\frac{1}{w^{*}}}=\frac{w^{*}+v^{*}+u^{*} v^{*} w^{*}}{1+w^{*} v^{*}+u^{*} w^{*}} \leq v^{*} .
$$


Case (7) $(u \leq 1, v \geq 1, w \geq 1, u+w \geq 1)$. Similarly, we have

$$
x_{n}^{*}=\frac{1+\frac{1}{u^{*}} v^{*}+v^{*} w^{*}}{w^{*}+v^{*}+\frac{1}{u^{*}}}=\frac{u^{*}+v^{*}+u^{*} v^{*} w^{*}}{1+u^{*} v^{*}+u^{*} w^{*}} \leq v^{*} .
$$

Case (8) $(u \geq 1, v \geq 1, w \geq 1, u+w \geq 1)$. By the same way, we have

$$
x_{n}^{*}=\frac{1+u^{*} v^{*}+v^{*} w^{*}}{u^{*}+v^{*}+w^{*}} \leq v^{*} .
$$

Inequalities (2.6)-(2.13) suggest our claim.

Remark. In fact, by Lemma 1 and in view of $u^{*} \geq 1$ and $w^{*} \geq 1$, the result $x_{n}^{*} \leq u^{*}, x_{n}^{*} \leq w^{*}$ can also be derived from the argument for front eight different cases.

Now let $X_{n}=\max _{n-l \leq i \leq n-1}\left\{x_{i}^{*}\right\}_{\text {for }}$ all $n \geq l$. By Lemma 2, we can deduce the following consequence.

Lemma 3. The sequence $\left\{X_{i}\right\}$ is monotonically non-increasing in $i$ which is much greater than $l$.

Since $X_{i} \geq 1$ for $i \geq l$, Lemma 3 implies that as $i$ tends to infinity, the sequence $\left\{X_{i}\right\}$ convergence to some limit, denote $X$, where $X \geq 1$.

\section{Convergence of solutions}

In what follows, we state and prove our main result in the sequence space.

Theorem 1. Suppose the initial data of equation $(1.1)\left(x_{-l}, x_{-l+1}, \ldots, x_{-1}\right) \in \mathbb{R}_{+}^{l}$. Then the solution sequence $\left\{x_{i}\right\}$ converges to the unique positive equilibrium $\bar{x}=1$.

Proof. Note that it suffices to show that the transformed sequence $\left\{x_{i}^{*}\right\}$ converges to 1. By the definition of $X_{i}$, the values of $X_{i}$ are taken on by entries in the sequence $\left\{x_{i}^{*}\right\}$, and as well, by Lemma $2,\left\{x_{i}^{*}\right\} \in\left[1, X_{i}\right]$ for $i \geq m$. Suppose $X>1$, then for any $\varepsilon \in(0$, $X)$, we can find an $N$ such that $\left\{x_{N}^{*}\right\} \in[X, X+\varepsilon]$, and for $i \geq N-l,\left\{x_{i}^{*}\right\} \in[1, X+\varepsilon]$.

Next we consider the eight possible cases again, and show that $X=1$. From the definitions of $x_{i}^{*}, X_{i}$ and $X$, the result follows.

Case (1) $(u \leq 1, v \leq 1, w \geq 1, u+w \geq 1)$. Here, by lemma 1 , we have

$$
X \leq x_{n}^{*}=\frac{\frac{1}{u^{*}}+\frac{1}{v^{*}}+w^{*}}{1+\frac{1}{u^{*}} \frac{1}{v^{*}}+\frac{1}{v^{*}} w^{*}} \leq \frac{1+\frac{1}{X+\varepsilon}+X+\varepsilon}{2+\frac{1}{X+\varepsilon}} .
$$

Hence

$$
\begin{aligned}
& 2 X^{2}+2 X \varepsilon+X \leq(1+X+\varepsilon)(X+\varepsilon)+1, \\
& \Rightarrow X^{2} \leq 1+\varepsilon+\varepsilon^{2} .
\end{aligned}
$$

Case (2) $(u \geq 1, v \leq 1, w \leq 1, u+w \geq 1)$. The argument is identical to that in Case (1).

Case (3) $(u \geq 1, v \leq 1, w \geq 1, u+w \geq 1)$. Here,

$$
X \leq x_{n}^{*}=\frac{u^{*}+\frac{1}{v^{*}}+w^{*}}{1+u^{*} \frac{1}{v^{*}}+\frac{1}{v^{*}} w^{*}} \leq \frac{2(X+\varepsilon)+\frac{1}{X+\varepsilon}}{3} .
$$


Therefore

$$
\begin{gathered}
3 X^{2}+3 X \varepsilon \leq 2(X+\varepsilon)^{2}+1 \\
\Rightarrow\left(X-\frac{\varepsilon}{2}\right)^{2} \leq 1+\frac{9}{4} \varepsilon^{2}, \\
\Rightarrow X \leq \sqrt{1+\frac{9}{4} \varepsilon^{2}}+\frac{\varepsilon}{2} .
\end{gathered}
$$

Case (4) $(u \leq 1, v \leq 1, w \leq 1, u+v \leq 1)$. Here,

$$
X \leq x_{n}^{*}=\frac{\frac{1}{u^{*}}+v^{*}+\frac{1}{w^{*}}}{1+\frac{1}{u^{*}} v^{*}+v^{*} \frac{1}{w^{*}}} \leq \frac{\frac{2}{X+\varepsilon}+X+\varepsilon}{3} .
$$

From this, we have

$$
\Rightarrow(X+\varepsilon)^{2} \leq 1+\frac{9}{16} \varepsilon^{2} .
$$

Namely,

$$
X \leq \sqrt{1+\frac{9}{16} \varepsilon^{2}}-\frac{\varepsilon}{4} .
$$

Case (5) $(u \leq 1, v \leq 1, w \leq 1, u+w \leq 1)$. We have

$$
X \leq x_{n}^{*}=\frac{1+\frac{1}{u^{*}} \frac{1}{v^{*}}+\frac{1}{v^{*}} \frac{1}{w^{*}}}{\frac{1}{u^{*}}+\frac{1}{v^{*}}+\frac{1}{w^{*}}} \leq \frac{1+\left(\frac{1}{X+\varepsilon}\right)^{2}+\left(\frac{1}{X+\varepsilon}\right)^{2}}{\frac{3}{X+\varepsilon}}
$$

which also implies

$$
X \leq \sqrt{1+\frac{9}{16} \varepsilon^{2}}-\frac{\varepsilon}{4} .
$$

Case (6) $(u \geq 1, v \geq 1, w \leq 1, u+w \geq 1)$. Here,

$$
X \leq x_{n}^{*}=\frac{1+u^{*} v^{*}+v^{*} \frac{1}{w^{*}}}{u^{*}+v^{*}+\frac{1}{w^{*}}} \leq \frac{1+(X+\varepsilon)^{2}+(X+\varepsilon)}{1+2(X+\varepsilon)}
$$

We have

$$
\begin{gathered}
2 X(X+\varepsilon)+X \leq 1+(X+\varepsilon)^{2}+(X+\varepsilon) \\
\Rightarrow X^{2} \leq 1+\varepsilon+\varepsilon^{2}
\end{gathered}
$$


Case (7) $(u \leq 1, v \geq 1, w \geq 1, u+w \geq 1)$. Here, it follows

$$
X \leq x_{n}^{*}=\frac{1+\frac{1}{u^{*}} v^{*}+v^{*} w^{*}}{w^{*}+v^{*}+\frac{1}{u^{*}}} .
$$

By the same argument with Case(6), we have

$$
X^{2} \leq 1+\varepsilon+\varepsilon^{2}
$$

Case (8) $(u \geq 1, v \geq 1, w \geq 1, u+w \geq 1)$. It here derives

$$
X \leq x_{n}^{*}=\frac{1+u^{*} v^{*}+v^{*} w^{*}}{u^{*}+v^{*}+w^{*}} \leq \frac{1+2(X+\varepsilon)^{2}}{3(X+\varepsilon)} .
$$

Hence

$$
X \leq \sqrt{1+\frac{9}{4} \varepsilon^{2}}+\frac{\varepsilon}{2} .
$$

Collecting all above inequalities which imply $X=1$ since $\varepsilon>0$ is arbitrary, we complete the proof. $\square$

\section{Generalization}

As mentioned above, the global asymptotic stability of positive solutions to the various equation listed above suggests that the same potentially holds for similar rational equations. We can deduce the following natural generalization of (1.1) and (1.4).

Corollary. Let $s \in \mathbb{N}^{+}$and $Z_{s}$ denote the set $Z_{s}=\{1,2, \ldots, s\}$. Suppose that $\left\{x_{i}\right\}$ satisfies the form

$$
x_{n}=\frac{\sum_{\substack{i=1 \\ i=1}}^{s} x_{n-k_{i}} x_{n-k_{i}} x_{n-k_{j}}+1}{i \neq j, j \in Z_{s}}, \quad n=0,1, \ldots
$$

with initial value $x_{-k}, x_{-k+1}, \ldots, x_{-1} \in \mathbb{R}_{+}$, here $k=\max _{1 \leq i \leq s}\left\{k_{i}\right\}$. Then the sequence $\left\{x_{i}\right\}$ converges to the unique equilibrium 1 .

Remark. If we consider the equation which is added a constant $a$ onto numerator and denominator of (4.1), the result is still viable. Indeed this corollary covers the results in [6].

Moreover, consulting the results of article $[6,7,10]$, by the similar way to Lemma 2, we have the following generalization.

Theorem 2. Suppose $f\left(x_{n-k_{1}}, \ldots, x_{n-k_{r}}\right) \in C\left(\mathbb{R}_{+}^{r}, \mathbb{R}_{+}\right), g\left(x_{n-m_{1}}, \ldots, x_{n-m_{s}}\right) \in C\left(\mathbb{R}_{+}^{s}, \mathbb{R}_{+}\right)$ and $h\left(x_{n-l_{1}}, \ldots, x_{n-l_{t}}\right) \in C\left(\mathbb{R}_{+}^{t}, \mathbb{R}_{+}\right)$satisfying $\left[g\left(x_{n-m_{1}}, \ldots, x_{n-m_{s}}\right)\right]^{*} \leq x_{n-m_{1}}^{*}$. Then the equation

$$
x_{n+1}=\frac{f+g+h}{f g+g h+1}, \quad n=0,1,2, \ldots .
$$


with the corresponding positive initial data has a unique positive equilibrium $\bar{x}=1$, and every solution of (4.2) converges to this point.

Proof. Let $\left\{x_{n}\right\}_{n=-p}^{\infty}$ be a solution sequence of equation (4.2) with initial data $x_{-p}, x_{-p+1}$, $x_{0} \in \mathbb{R}_{+}$, where $p=\max \left\{k_{r}, m_{s}, l_{t}\right\}$. By the definition of $x_{n}^{*}$, From the equation (4.2), the arguments in Lemma 2 and the hypothesis, it follows that for any $n \geq 0$,

$$
1 \leq x_{n+1}^{*}=\left[\frac{f+g+h}{f g+g h+1}\right]^{*} \leq\left[g\left(x_{n-m_{1}}, \ldots, x_{n-m_{s}}\right)\right]^{*} \leq x_{n-m_{1}}^{*}
$$

from which we get that for any $n \geq 0$ and $0 \leq i \leq m_{1}, 1 \leq x_{i+(n+1)\left(m_{1}+1\right)}^{*} \leq x_{i+n\left(m_{1}+1\right)}^{*}$. Hence the sequence $\left\{x_{i+n\left(m_{1}+1\right)}^{*}\right\}_{n=0}^{\infty}$ with $0 \leq i \leq m_{1}$ is convergent. Denote the limit as $\lim _{n \rightarrow \infty} x_{i+n\left(m_{1}+1\right)}^{*}=A_{i}$, then $A_{i} \geq 1$. Write $M=\max \left\{A_{0}, A_{1}, \ldots, A_{m_{1}}\right\}$ and $A_{i+n\left(m_{1}+1\right)}=A_{i}$ for any integer $n$. Then there exists some $0 \leq j \leq m_{1}$ such that $\lim _{n \rightarrow \infty} x_{j+n\left(m_{1}+1\right)}^{*}=M$. From (4.3), it suggests $M=g\left(M, A_{j-1-m_{2}}, \ldots, A_{j-1-m_{s}}\right)=M$.

Combining the facts $1+a b \geq a+b$ and $\frac{a b+1+b c}{a+b+c} \leq \frac{a b+1+b c+a b c}{a+b+c+a c}$, where $a \geq 1, b \geq 1$ and $c$ $\geq 1$, for the different situation in Theorem 1 , we have

$$
x_{n+1}^{*} \leq \begin{cases}\frac{f^{*}+g^{*}+f^{*} g^{*} h^{*}}{1+f^{*} g^{*}+f^{*} h^{*}} \leq \frac{f^{*}+g^{*}+f^{*} g^{*} h^{*}}{f^{*}+g^{*}+f^{*} h^{*}}, & \text { for Case }(1,2,6,7), \\ \frac{f^{*} g^{*}+1+g^{*} h^{*}}{f^{*}+g^{*}+h^{*}} \leq \frac{f^{*} g^{*}+1+g^{*} h^{*}+f^{*} g^{*} h^{*}}{f^{*}+g^{*}+h^{*}+f^{*} h^{*}}, & \text { for Case }(3,8), \\ \frac{f^{*}+h^{*}+f^{*} g^{*} h^{*}}{g^{*} h^{*}+f^{*} g^{*}+f^{*} h^{*}} \leq \frac{f^{*}+h^{*}+f^{*} g^{*} h^{*}}{f^{*}+h^{*}+f^{*} h^{*}}, & \text { for Case }(4,5) .\end{cases}
$$

Therefore

$$
1 \leq M \leq \begin{cases}\frac{f^{*}+M+f^{*} M h^{*}}{f^{*}+M+f^{*} h^{*}}, & \text { for Case }(1,2,6,7), \\ \frac{f^{*} M+1+M h^{*}+f^{*} M h^{*}}{f^{*}+M+h^{*}+f^{*} h^{*}}, & \text { for Case }(3,8), \\ \frac{f^{*}+h^{*}+f^{*} M h^{*}}{f^{*}+h^{*}+f^{*} h^{*}}, & \text { for Case }(4,5),\end{cases}
$$

from which it follows $M=1$. This implies $A_{i}=1$ for $0 \leq i \leq m_{1}$ and $\lim _{n \rightarrow \infty} x_{n}^{*}=1$. Since $1 / x_{n}^{*} \leq x_{n} \leq x_{n}^{*}$, we obtain $\lim _{n \rightarrow \infty} x_{n}=1$. ㅁ

Remark. The stability of solution to equation (4.2) is ever proposed to consider as a conjecture by K.S.Berenhaut etc. in [7]. Indeed, Theorem 1 proved the conjecture partially.

In addition, gathering lots of relevant work listed in reference, we put forward the following conjecture.

Conjecture. Let $s \in \mathbb{N}^{+}, Z_{s}=\{1,2, \ldots, s\}$ and $l_{i j} \geq 0$. Suppose that $\left\{x_{i}\right\}$ satisfies

$$
x_{n}=\frac{\sum_{j=1}^{s} \prod_{i \in Z_{s}} x_{n-k_{i j}}^{l_{i j}}}{\sum_{j=1}^{s-1} \prod_{i \in Z_{s}} x_{n-k_{i j}}^{l_{i j}}+1}, \quad n=0,1,2, \ldots
$$

with $x_{-k}, x_{-k+1}, \ldots, x_{-1} \in \mathbb{R}_{+}, k=\max _{i, j \in Z_{s}}\left\{k_{i, j}\right\}$, then the sequence $\left\{x_{i}\right\}_{i=0}^{\infty}$ converges to the unique equilibrium 1 . 
Acknowledgements

The authors would like to thank the reviewers and the editors for their valuable suggestions and comments; The authors wish to express their deep gratitude to Professor C.Y. Wang for his valuable advice and constant encouragement for this work supported in part by Natural Science Foundation for Colleges and Universities in Hebei Province(Z2011111, Z2011162) and Human Resources and Social Security Subject of Hebei Province(JRS-2011-1042).

\section{Authors' contributions}

QS completed the main part of this paper, QX and GY corrected the main theorems. XL participated in the design and coordination. All authors read and approved the final manuscript.

\section{Competing interests}

The authors declare that they have no competing interests.

Received: 17 May 2011 Accepted: 27 September 2011 Published: 27 September 2011

\section{References}

1. Li, W., Sun, H.: Global attractivity in a rational recursive sequence. Dyn. Syst. Appl. 11, 339-346 (2002)

2. Kocic, VL, Ladas, G: Global Behavior of Nonlinear Difference Equations of Higher Order with Application. Kiuwer Academic Publishers, Dordrecht. (1993)

3. Kulenovic, MR., Ladas, G: Dynamics of Second Order Rational Difference Equations with Open Problems and Conjectures. Chapman Hall/CRC, Boca Raton. (2001)

4. Çinar, C: On the positive solutions of the difference equation $x_{n+1}=\frac{x_{n-1}}{1+x_{n} x_{n-1}}$. Appl. Math. Comput. 150, $21-24$ (2004). doi:10.1016/50096-3003(03)00194-2

5. Yang, X, Su, W, Chen, B, Megson, GM, Evans, DJ: On the recursive sequence. Appl. Math. Comput. 162, 1485-1497 (2005). doi:10.1016/j.amc.2004.03.023

6. Li, X: Global behavior for a fourth-order rational di_erenceequation. J. Math. Anal. Appl. 312, 103-111 (2005)

7. Berenhaut, KS, Foley, JD, Stevic, S: The global attractivity of the rational difference equation $y_{n}=\left(y_{n-k}+y_{n-m}\right)>/\left(1+y_{n-}\right.$ $y_{n-m}$ ). Appl. Math. Lett. 20, 54-58 (2007). doi:10.1016/j.aml.2006.02.022

8. Papaschinopoulos, G, Schinas, CJ: Global asymptotic stability and oscillation of a family of difference equations. J. Math. Anal. Appl. 294, 614-620 (2004). doi:10.1016/j.jmaa.2004.02.039

9. Sun, T., Xi, H.: Global attractivity for a family of nonlinear difference equations. Appl. Math. Lett. 20, 741-745 (2007). doi:10.1016/.j.aml.2006.08.024

10. Li, Z., Zhu, D.: Global asymptotic stability of a higher order nonlinear difference equation. Appl. Math. Lett. 19, 926-930 (2006). doi:10.1016/j.aml.2005.10.014

doi:10.1186/1687-1847-2011-36

Cite this article as: Shi et al:: Dynamic behavior of a nonlinear rational difference equation and generalization.

Advances in Difference Equations 2011 2011:36.

\section{Submit your manuscript to a SpringerOpen ${ }^{\circ}$ journal and benefit from:}

- Convenient online submission

- Rigorous peer review

- Immediate publication on acceptance

- Open access: articles freely available online

- High visibility within the field

- Retaining the copyright to your article

Submit your next manuscript at $\gg$ springeropen.com 